REVIEW ESSAY

Jonathan Evans, St.B.T. Thinking and Reasoning: A Very Short Introduction.

Oxford: Oxford University Press, 2017. 134 pages.

Jack Lyons and Barry Ward. The New Critical Thinking: An Empirically Informed Introduction.

New York: Routledge, 2017. 380 pages.

Stuart Hanscomb. Critical Thinking: The Basics.

New York: Routlege, 2016. 260 pages.

Galen A. Foresman, Peter S. Fosl, and Jamie C. Watson. The Critical Thinking Toolkit.

\title{
CRITICAL THINKING ABOUT CRITICAL THINKING: RECENT BOOKS ON THINKING AND REASONING
}

\author{
Noelle Leslie dela Cruz
}

Reason is often thought to be the distinguishing characteristic of our species. While scientists recognize that animals can make inferences based on previous information, humans are unique in that we may be conscious of a great deal of our inferential processes. Furthermore, we have the capacity for imagination or innovative thinking. However, rationality is not without its paradoxes. While it underpins the achievements of science and technology, securing a dominant status for our species, human reasoning is also prone to error. Cognitive biases belie the idea that human thought follows the rules of logic. This has led to the so-called "rationality wars," which revolve around the question of whether we are rational or irrational, and what 
"rationality" ultimately means.

Four notable books on the subject of thinking and reasoning have recently been released, shedding light on the issue. The first is Jonathan St. B.T. Evans' pithy work, Thinking and Reasoning: A Very Short Introduction. The other three books are less general in scope but more detailed in the treatment of their subject matter, which is critical thinking. The psychology of thinking, of which Evans provides a useful précis, is related to the interdisciplinary field of critical thinking, which grew out of education. The current thinking about thinking — which involves what psychologists call dualprocess theories - has influenced recent books about critical thinking. Their starting point is the notion that there are two mental processes, one intuitive and the other rational. Type 1 reasoning, which is quick and efficient when employed in a wide array of activities, is nonetheless prone to cognitive errors. Type 2 reasoning, meanwhile, is slower and more deliberate. Inasmuch as the latter is susceptible to conscious training, it is the focus of recent books about critical thinking. Thus, in light of recent psychological findings, tried-and-true topics such as arguments and evaluating arguments, informal fallacies, evaluating claims, and the rules of debate, are presented in a new, "empirically informed" manner.

Evans' book, which is part of the Very Short Introductions series of Oxford University Press, is not a book about critical thinking, though it lays down the more foundational developments in the psychology of thinking. It opens with a definition of thinking as the ability to imagine and ask the right questions, i.e. to see things from a new perspective (1). It then provides a concise history of the psychology of thinking, from the introspective method pioneered by William James and Sir Francis Galton, to Freudian theory and the now much-derided behaviorism of B.F. Skinner, concluding with cognitive psychology, currently the most widely accepted view. One paradigm of thinking tends to be supplanted by another as psychological theories come and go. For instance, the theory of the unconscious led to the eventual rejection of introspection as necessarily limited to reflective awareness. Meanwhile, the apparent unfalsifiability of the Freudian unconscious led to the behaviorist impulse to reject the idea of "mind" altogether, focusing on verifiable phenomena such as stimulus and response. Finally, in the contemporary digital era, the mind is viewed in computational terms. Accordingly, cognitive psychologists study the brain as a kind of high-level processor of information.

At least four distinct mental processes, or four major features of intelligence, are discussed, namely: problem-solving, thinking hypothetically, decision-making, and finally, deductive and statistical reasoning. Evans notes that "the solution of novel problems is what generally marks our species out as different from both animals and earlier hominids" (18). Humans also have an additional characteristic, at least in comparison to artificially intelligent machines: we can solve even ill-definedproblems, or problems whose parameters are not neatly fixed. On the other hand, thinking hypothetically involves the ability to imagine how things may turn out in the future, or how they could have turned out differently in the past. Our capacity for constructing complex mental models underlies such life-saving endeavors as the making of medical diagnoses as well as scientific thinking itself. Meanwhile, the psychology of decision-making has greatly influenced the field of economics, which in turn is premised on the 


\section{NOELLE LESLIE DELA CRUZ}

idea of human rationality when it comes to expected value and utility. Finally,deductive reasoning and statistical inference, which follow normative rules, allow us to reach conclusions that range from highly probable to necessary. Our certainty in our conclusions about the world renders it appreciatively more livable.

Despite these feats of rationality, however, a growing body of empirical research indicates that human beings are also prone to cognitive biases. These cognitive biases throw into question the validity of normative theories of rationality, such as decision theory, probability theory, and logic. Laboratory experiments - such as the well-known Wason selection task - suggest that human reasoning does not quite follow normative rules. Asked to test the veracity of the conditional statement, "If there is an A on one side of the card, then there is a 3 on the other side of the card," test subjects tend to turn over intuitively appealing, through ultimately incorrect, cards (39). Meanwhile, such phenomena as framing, omission bias, and focusing bias, to name a few, may lead to irrational decision-making (54-58). These findings have fueled what Evans calls "the great rationality debate," in which key thinkers committed to the idea of human rationality question the methods and contexts of such empirical findings, while some others call for a redefinition of rationality in non-normative terms. Evans' concluding chapter discusses dual-process theories, which offer a provisional solution to the rationality problem. Type 1 thinking is associated with the power of intuition, while Type 2 thinking is associated with intelligence and working memory. This theory explains cognitive biases revealed in laboratory settings, because in such settings, "the typical laboratory tasks are designed to require Type 2 processing for their solution and generally to preclude relevant prior experience that could provide helpful Type 1 intuitions" (115).

Recent books on critical thinking, such as The New Critical Thinking: An Empirically Informed Introduction by Jack Lyons and Barry Ward, and Critical Thinking: The Basics by Stuart Hanscomb, apply these findings from psychology, in particular dual-process theories. Lyons and Ward incorporate in their framework what they call the Dual Systems Theory of the brain, focusing on the training and improvement of System 2 (7). They also incorporate the influence of cognitive biases in their discussion of how to evaluate arguments. Meanwhile, Hanscomb devotes his first chapter entirely to "Rationality, Cognitive Biases, and Emotions," which deals with a near-tocomprehensive list of psychological errors such as the confirmation bias; the selfserving bias; cognitive dissonance, commitment, and consistency; the representativeness heuristic; anchoring and contrast effect; authority and likeability; and social proof. While it is Lyons and Ward who claim to have written about "the new critical thinking," it is actually Hanscomb who provides the more psychologically informed material. A third book on critical thinking, The Critical Thinking Toolkit by Galen A Foresman, Peter S. Fosl, and Jamie C. Watson, also incorporates insights and practices from psychology. Accordingly, they devote Chapter Seven, entitled "Tools for Critical Thinking about Experience and Error," to strategies for identifying and correcting cognitive and environmental errors. Even this chapter provides more empirical information about thinking than does the entire book of Lyons and Ward, though it must be noted that the latter has its own strengths, if not in terms of what it touts as "new" in its take on critical thinking. 
All three books cover the essential components of critical thinking, though they tend to differ in their objectives and special features. Thus, all of them may be used without much overlap in any course on critical thinking. The New Critical Thinking focuses on one's assessment of the reasoning, argument, and persuasion by others, in what the authors call the "consumer's view" rather than the producer's (1). By contrast, Critical Thinking: The Basics not only aims to improve the reader's ability to reason and argue, but also his or her ability to evaluate the arguments of others. In this sense, the latter is more comprehensive than the former. Meanwhile, The Critical Thinking Toolkit provides the broadest approach, situating the principles of good argumentation in the context of a variety of disciplines, including philosophy, psychology, literature, and social and political science (2).

Though they feature the greatest hits of any critical thinking course, as it weresuch as (re-)constructing and evaluating arguments, informal fallacies, evaluating claims, and the rules of rational dialogue - the books present and connect these topics differently, to differing effects. Thus, depending on the instructor's or student's purposes, a particular book may be preferable to the others.

For example, Lyons and Ward follow a traditional division into deductive and inductive reasoning, which allows for a finely tuned analysis of the various forms of thinking under each category. In addition to logical validity, they cover statistical syllogism, inductive generalization, analogical arguments, inferences to the best explanation, causal inferences, and probabilities and inferences. The section on evaluating premises is broadly divided into "testimony" and "science," after which there is a final section on argumentation, itself divided into "rhetoric" (i.e. the informal fallacies) and "dialectic" (i.e. the rules of debate). An important insight from this section is how our adversaries are really our allies in the process of learning and dialoguing (327). If you are looking for an exercise-intensive workbook that covers a wide range of argument-evaluation strategies, this book is a good choice.

Meanwhile, Hanscomb's book, rather than pursuing the deductive-inductive dichotomy, focuses on "plausible arguments," also known as "presumptive" or "defeasible" arguments, following the work of Douglas Walton. This highly pragmatic type of argument characterizes everyday decision-making dialogues. It has three characteristics: (1) It makes claims based on what is reasonably expected in familiar situations; (2) it shifts the burden of proof to any claim that contradicts the one being made; and (3) it recognizes that the conclusion drawn is provisional in nature. The main body of the book is thus divided into three broad types of plausible arguments, namely: arguments about social power; causal arguments, generalizations, and arguments from consequences; and arguments from analogy. In his analysis of each type, Hanscomb incorporates a discussion of the rhetorical force of the associated fallacies, the cognitive biases that lead to them, their relationship with critical thinking dispositions, and their impact on rational dialogues. This ingenious format ensures that the reader clearly sees the connections among the different components of critical thinking, from the evaluation of arguments to the critique of fallacies to the recognition of psychological errors to our responsibility as critical thinkers engaged in rational discussion. Indeed, the book connects ethical living with critical thinking, opening with a chapter on "critical thinking 


\section{NOELLE LESLIE DELA CRUZ}

dispositions" or "epistemic virtues" that serve as overarching themes. These include love of truth, open-mindedness, flexibility, modesty, self-knowledge, meta-cognition, and dialogical dispositions such as courage, staying focused, and respect for others. Critical Thinking: The Basics is a book true to the spirit of a Socrates or a Bertrand Russell. It presents critical thinking not only as an intellectual activity, but also as a path toward wisdom.

Finally, The Critical Thinking Toolkit is by far the most philosophically oriented. In fact, the authors encourage its use alongside two other books in the Wiley's Toolkit series, i.e. The Philosopher's Toolkit and The Ethics Toolkit. These handy books present broadly philosophical concepts in a pragmatic rather than a historical manner. The emphasis is on how such concepts may be employed in solving philosophical problems. Thus, Foresman et. al.'s book frames the components of critical thinking in terms of "tools," e.g. "Tools for Critical Thinking about Experience and Error," "Tools for Critical Thinking about Science," "Tools for Deductive Reasoning," "Tools for Critical Thinking about Induction," and "Tools for Detecting Informal Fallacies." Its chapters on deductive argumentation emphasize the role of formal logic, and thus include a comprehensive discussion of both categorical logic and truth functional logic. While the book lacks a discussion of the rules of dialogue or debate, which are a staple of any critical thinking textbook, it does include a couple of chapters that are not ordinarily found in such a textbook. One is the chapter on "Tools for Critical Thinking about Justification," which is none other than a readable overview of what's at stake in contemporary epistemology. Another is the chapter on "Tools from Rhetoric, Critical Theory, and Politics," which extracts what is useful for critical thinking from such diverse lenses of analysis as metanarratives, tropes, semiotics, media studies, deconstruction, feminism, cultural critique, race and class critiques, historicist critiques, and ecological critiques. These are broad areas of thought, indeed, and the extraordinary achievement of the Toolkit is its demonstration that all of these approaches are themselves forms of critical thinking. 